\title{
Simple potential bioanalysis micro reactor fabricated by vertically-aligned multi-walled carbon nanotubes (MWCNTS)
}

\begin{abstract}
A simple Microreactor was fabricated by vertically-aligned multi-walled carbon nanotubes (MWCNTs) on the mc-Si substrate. MWCNTs were synthesized by homemade equipment aided with chemical vapor deposition (CVD). The wettability of the surfaces of Microreactor with the synthesized MWCNTs, Au coating and plasma irradiation were investigated, respectively. Results show that the initial surface with the MWCNTs film behaves as a super-hydrophobic surface. However, it changes to a super-hydrophilic surface after coated with $\mathrm{Au}$ or irradiated by plasma. It shows that Microreactor with treated MWCNTs film can be used as potential applications for bioanalysis.
\end{abstract}

Keywords: bioanalysis microreactor, wettability, hydrophobic, hydrophilic, plasma, x-ray lithography
Volume 3 Issue 2 - 2017

\author{
Kelvii Wei Guo, Hon Yuen TAM \\ Department of Mechanical and Biomedical Engineering, City \\ University of Hong Kong, Hong Kong
}

Correspondence: Kelvii Wei Guo, Department of Mechanical and Biomedical Engineering, City University of Hong Kong, 83 Tat Chee Avenue, Kowloon Tong, Kowloon, Hong Kong, Email kelviiguo@yahoo.com

Received: October 18, 2016 | Published: May 22, 2017
Abbreviations: MWCNTs, multi-walled carbon nanotubes; CVD, chemical vapor deposition; LOC, lab-on-a-chip; $\mu$ TAS, micro-total analysis systems; SEM, scanning electron microscopy; TEM, transmission electron microscopy; ECR, electron cyclotron resonance;

\section{Introduction}

Nanomaterials are playing the more and more important role in electronic, biological, chemical and medical applications. In particular, carbon nanotubes (CNTs) have been attracting significant attention because of their unique characteristics. ${ }^{1} \mathrm{CNT}$ electrodes and CNT-based reactors have been applied as electrodes in fuel cells, ${ }^{2,3}$ and reaction fields in biosensors, ${ }^{4,5}$ etc. The development of labon-a-chip (LOC) devices, a term for "micro-total analysis systems ( $\mu$ TAS)", for biochemical analyses has seen explosive growth over the past decade. ${ }^{6-8}$ LOC devices are now widely used in biology and biotechnology, ${ }^{9}$ DNA and proteins analysis, ${ }^{10,11}$ high throughput screening ${ }^{12}$ and chemical reactions. ${ }^{13}$ Various bio-substances are often analyzed by detecting the immune reaction between antigens in a sample solution or antibodies immobilized on micro channel surfaces using fluorescence detection and the surface Plasmon resonance method. In such applications, detection depends significantly on the contact surface. The design and creation of surfaces that have high surface per volume is currently one of the important issues in LOC and $\mu$ TAS. Aim to develop a new simple Microreactor for bioanalysis, the high density vertically-aligned MWCNTs were synthesized by homemade equipment aided with the chemical vapor deposition (CVD) and studied by scanning electron microscopy (SEM) and transmission electron microscopy (TEM). Also, the wettability of the MWCNTs was investigated after $\mathrm{Au}$ coating and plasma irradiation by electron cyclotron resonance (ECR) for potential bioanalysis applications.

\section{Concept of fabricated 3D surface by vertically-aligned MWCNTs for micro reactor based $\mu$ TAS}

Since CNTs have high-aspect ratios, in micro total analysis systems ( $\mu \mathrm{TAS})$, they can form a much wider reaction field on the surface, which is useful for biological applications. ${ }^{14}$ The micro reactors in these systems have been proposed to be micro channels or micro wells based on the droplets. All of these systems have surfaces with a very high surface-to-volume ratio. From this point-of-view, a reactor with MWCNTs can improve existing Microreactor capabilities obviously. In order to achieve a high quality $\mu \mathrm{TAS}$ reactor, synthesis of MWCNTs with a high density is required. Figure 1 shows the concept of a Microreactor with MWCNTs. The relevant proposed method is to locate primary antibodies on the CNTs surface. Thus, primary antibodies are located in three dimensions. Furthermore, the representative size ( $\mathrm{L}$ in the Figure 1) will be changed (cf. Figure 1A $\&$ 1B). In a traditional Microreactor, $\mathrm{L}$ is the depth or width of a micro channel or a micro well. However, $\mathrm{L}$ in the new reactor becomes the length between vertically aligned CNTs if the reaction field height is coincident with the CNT length. Consequently, the basic diffusion can be expressed as, where $\mathrm{D}$ is the diffusion coefficient and $t$ is the diffusion time. By decreasing the value of $\mathrm{L}, \mathrm{t}$ decreases as well. When the primary antibodies are located in three dimensions, appropriate decreases in the size will lead to higher sensitivities and reaction speed. It is well known that etching-based processes are used for the fabrication of $\mu \mathrm{m} \sim \mathrm{nm}$ size structures. The advantage of etching is that it can be used to fabricate structures with high accuracy. However, it is difficult for etching to make under several nanometers just like CNTs. UV and X-ray lithography can make structures or patterns within $100 \mathrm{~nm}$, but these methods are expensive. On the other hand, synthesis of CNTs currently can be achieved easily at low cost. In this research, MWCNTs are synthesized only by the catalyst pre-deposited 
by CVD and ethanol with the home-made equipment. Furthermore, CNTs unique structures such as high aspect ratios and densities cannot be fabricated by etching techniques. Therefore, the new Microreactor with CNTs proposed in this work will have a promising potential application in $\mu$ TAS for bioanalysis.

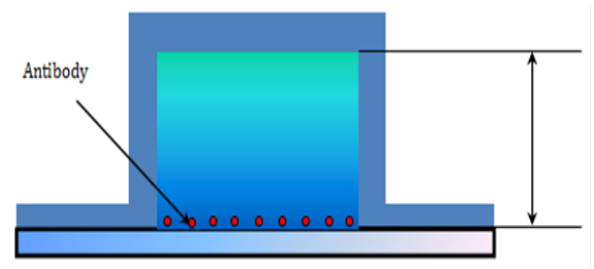

(A)

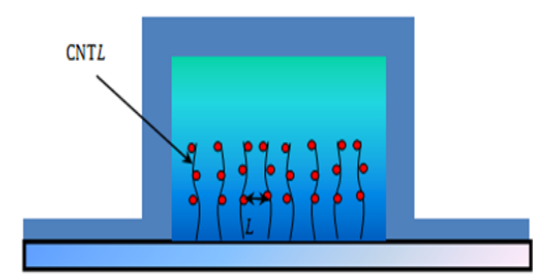

(B)

Figure I Concept of a microreactor based $\mu$ TAS with CNTs.

A. Traditional bioanalysis microreactor

B. New bioanalysis microreactor

\section{Experimental procedure}

\section{Catalyst coated for synthesizing vertically-aligned MWCNTs}

The catalyst $(\mathrm{Fe})$ was deposited on the substrate ( $\mathrm{mc}-\mathrm{Si}$ ) by electron cyclotron resonance (ECR) and the related deposition parameters are listed in Table 1.

Table I ECR processing parameters for Fe deposition

\begin{tabular}{ll}
\hline Parameters & \\
\hline Substrate & $\mathrm{Si}(100)$ \\
Catalyst & $\mathrm{Fe} / \mathrm{Al}$ \\
Irradiation time & $60,150,300,600 \mathrm{~s}$ \\
Accelerated voltage & $2,500 \mathrm{~V}$ \\
lon current density & $12.0 \mathrm{~mA} / \mathrm{cm}^{2}$ \\
Gas & $\mathrm{Ar}$ \\
Gas flow rate & $0.6 \mathrm{SCCM}$ \\
Vacuum & $1.5 \times 10^{-4} \mathrm{~Pa}$ \\
\hline
\end{tabular}

\section{MWCNTs synthesis}

For vertically-aligned MWCNTs synthesis, the schematic diagram of the home-made equipment is shown in Figure 2. The size of vacuum chamber and the heater is $\Phi 100 \times 150 \mathrm{~mm}$ and $50 \times 30 \mathrm{~mm}$ respectively. The electrical resistance is taken as the heating resource. The maximum temperature can be reached to $1680^{\circ} \mathrm{C}$. Ethanol in a ceramic container is located under the heater and the specimen deposited with the catalyst is placed on the heater in the vacuum chamber. A DC power supplier is applied to heat the specimens in the vacuum $\left(1 \times 10^{-2} \mathrm{~Pa}\right)$ under $38 \sim 40 \mathrm{~A}$.

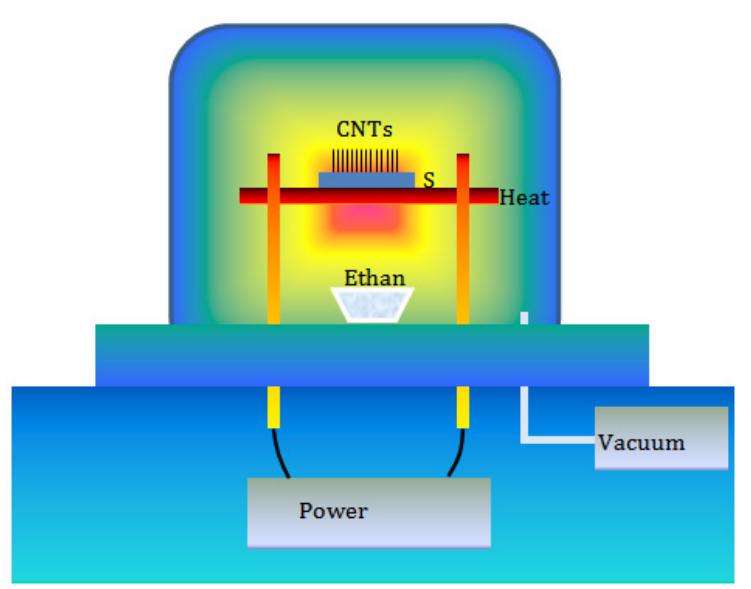

Figure 2 Schematic diagram of home-made equipment for MWCNTs.

\section{Raman spectroscopy analysis}

The characteristics of the vertically aligned MWCNTs synthesized were evaluated by Raman spectroscopy analysis. Table 2 shows the conditions of the Raman spectroscopy analysis. Generally, typical Raman shifts $\left(\mathrm{cm}^{-1}\right)$ of CNTs include the G-band, the D-band and RBM, where the peak of the G-band is at about $1590 \mathrm{~cm}^{-1}$. and the $\mathrm{D}$-band is $1350 \mathrm{~cm}^{-1}$. The CNTs is evaluated on the basis of the ratio between the G-band and the D-band.

Table 2 Conditions for Raman spectroscopy analysis

\begin{tabular}{ll}
\hline Conditions & \\
\hline Laser wavelength & $532 \mathrm{~nm}$ \\
Laser power & $30 \mathrm{~mW} \times 0.5 \%$ \\
Irradiation spot & $5 \mu \mathrm{m}$ \\
Integration times & 30
\end{tabular}

\section{Microreactor fabrication with CNTs}

Figure 3 shows the fabrication procedure for the Microreactor with MWCNTs, where unit reactor is machined by dice cutting after the catalyst deposition. Table 3 shows the corresponding parameters of dice cutting and Microreactor.

Table 3 Parameters of dice cutting and microreactor

\begin{tabular}{ll}
\hline Parameters & \\
\hline Size of Micro reactor unit & $100 \mu \mathrm{m} \times 100 \mu \mathrm{m}$ \\
Gap among reactors & $35 \mu \mathrm{m}$ \\
Cutting speed & $0.5 \mathrm{~mm} / \mathrm{s}$ \\
Cutting depth & $50 \mu \mathrm{m}$ \\
\hline
\end{tabular}




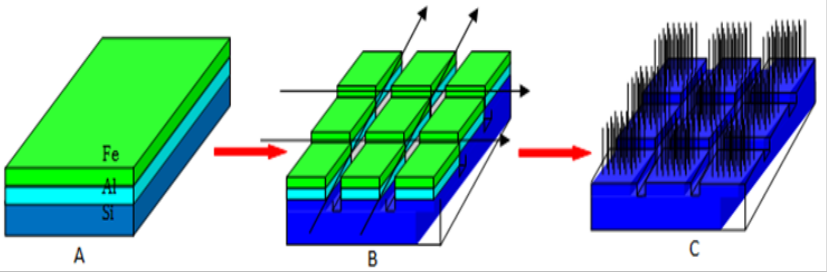

Figure 3 Miroreactor fabrication with MWCNTs.

A. Catalyst deposition.

B. Dicing patterns for microreactor.

C. Microreactor with MWCNTs.

\section{Results and discussion}

Morphology of synthesized vertically-aligned MWCNTs

The synthesized vertically-aligned MWCNTs are shown in Figure 4. It shows that the general growth direction was normal to the surface. They appeared wiggly due to CNTs tightly packed during growth. The TEM image of one typical CNT is shown in Figure 5. The image reveals that they were definitely multi-walled nanotubes. The diameter was about $3 \sim 5 \mathrm{~nm}$. Also, the lengths of the CNTs were estimated to be $10 \sim 35 \mu \mathrm{m}$. Moreover, the morphology of the relevant Microreactor is shown in Figure 6.

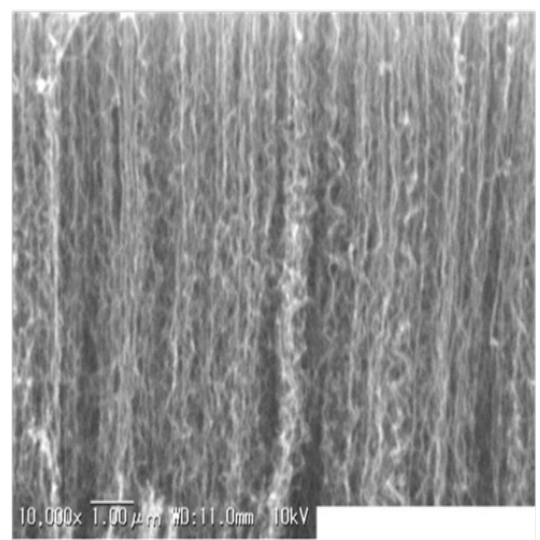

Figure 4 SEM image of synthesized vertically-aligned MWCNTs.

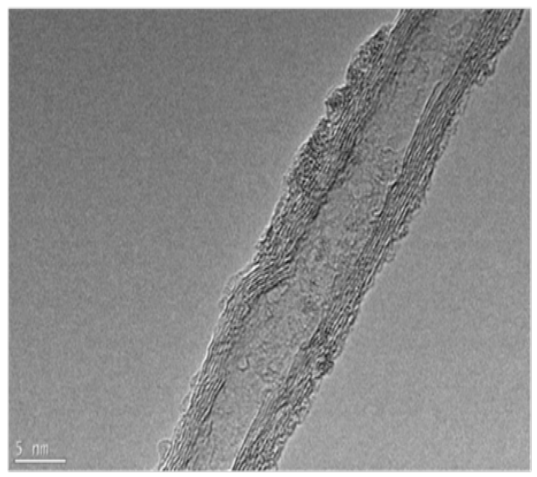

Figure 5 TEM image of a CNT.

\section{Result of raman spectroscopy analysis}

The result of Raman spectroscopy analysis of the synthesized vertically-aligned MWCNTs is shown in Figure 7. It elucidates that the peak of D-band was higher than that of G-band and the ratio of IG/ ID was about 0.75 , which indicates that many CNTs were disordered. As the media in the Microreactor, the disordered MWCNTs will be inclined to catch the antibody more easily. Therefore, the Microreactor with such MWCNTs must be more effective for the practical applications than that of the traditional reactors.

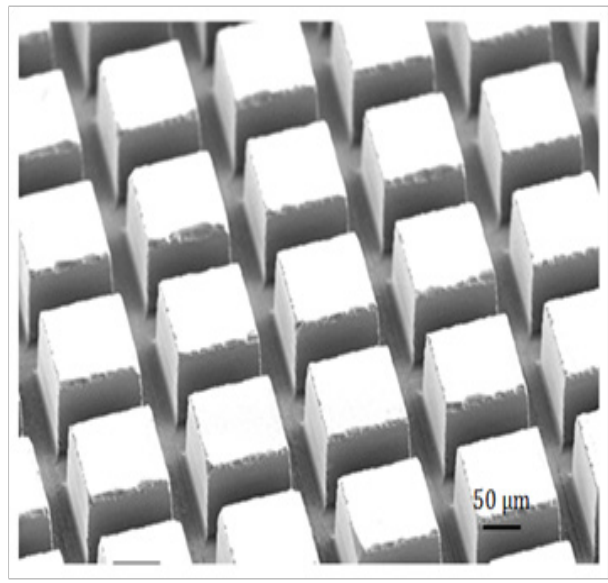

Figure 6 Microreactor with vertically-aligned MWCNTs.

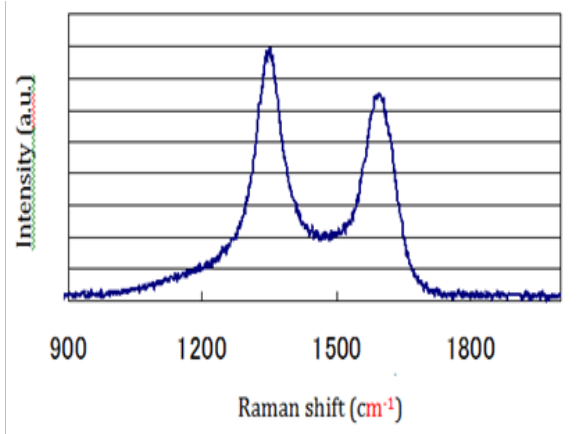

Figure 7 Raman spectra of synthesized MWCNTs.

\section{Wettability of the vertically-aligned MWCNTs}

The wettability of surface of Microreactor with MWCNTs was investigated by the droplet of pure water and the volume of the droplets was $1 \mu l$. Result shows that the contact angle on the verticallyaligned CNTs film was $146.6^{\circ}$ resulted in super-hydrophobic as shown in Figure 8A and the related schematic diagram is shown in Figure $8 \mathrm{~B}$. For changing the wettability, the Au coating and plasma irradiation were carried out with the plasma irradiation time of $5 \mathrm{~min}$ and ion current of $6.5 \mathrm{~mA}$. Figure 9 shows the contact angles of the vertically-aligned MWCNTs surface coated with Au and irradiated with the plasma, respectively. Results show that the processed surface changed to a super-hydrophilic surface after Au coating and plasma irradiation. More attractively, the contact angle on the CNTs film irradiated by plasma was close to $0^{0}$ as shown in Figure $9 \mathrm{C}$ and its corresponding schematic diagram is shown in Figure 9D. However, the contact angle of the vertically-aligned MWCNTs surface with $\mathrm{Au}$ coating (Figure 9A \& 9B) was higher than that of surface with plasma irradiation which indicates the droplet cannot be infiltrated into CNTs completely with Au coating on the surface resulted in the successive application failure. 


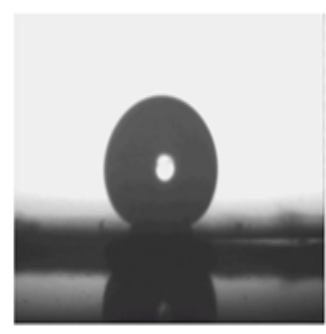

(A)

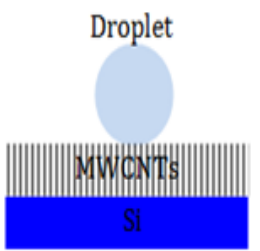

(B)

Figure 8 Wettability of the vertically-aligned MWCNTs.

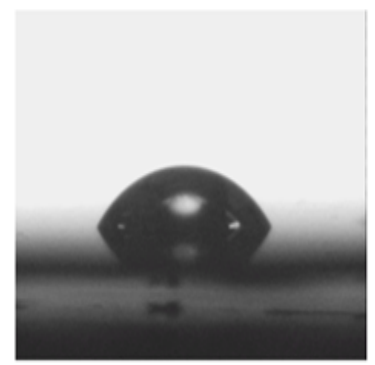

(A)

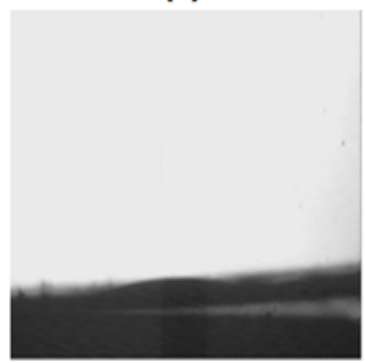

(C)

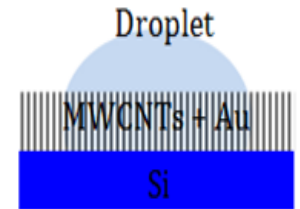

(B)

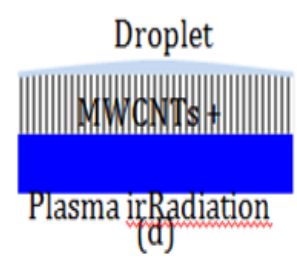

Figure 9 Wettability of the vertically-aligned MWCNTs film with Au coating and plasma irradiation.

\section{Conclusion}

The simple Microreactor potential for bioanalysis was successfully fabricated with MWCNTs. Results show that the initial synthesized surface with the MWCNTs film behaves as a super-hydrophobic surface. After coating with Au or irradiating by plasma it changes to a super-hydrophilic surface. Moreover, the effect of plasma treatment on the wettability changed from super-hydrophobic to superhydrophilic is definitely superior to that of Au coating. It indicates that Microreactor with surface treated MWCNTs can be more in favor of the potential applications for bioanalysis.

\section{Acknowledgements}

None.

\section{Conflict of interest}

The author declares no conflict of interest.

\section{References}

1. Shah KA, Tali BA. Synthesis of carbon nanotubes by catalytic chemical vapour deposition: A review on carbon sources, catalysts and substrates. Materials Science in Semiconductor Processing. 2016;41:67-82.

2. Zamani P, Higgins DC, Hassan FM, et al. Highly active and porous graphene encapsulating carbon nanotubes as a non-precious oxygen reduction electrocatalyst for hydrogen-air fuel cells. Nano Energy. 2016;26:267-275.

3. Moreno NG, Gervasio D, García AG, et al. Polybenzimidazole-multiwall carbon nanotubes composite membranes for polymer electrolyte membrane fuel cells. Journal of Power Sources. 2015;300:229-237.

4. Lawal AT. Synthesis and utilization of carbon nanotubes for fabrication of electrochemical biosensors. Materials Research Bulletin. 2016; $73: 308-350$.

5. Rabti A, Raouafi N, Merkoçi A. Bio (Sensing) devices based on ferrocene-functionalized graphene and carbon nanotubes. Carbon. 2016;108:481-514.

6. Lafleur JP, Jönsson A, Senkbeil S, et al. Recent advances in lab-ona-chip for biosensing applications. Biosensors and Bioelectronics. 2016;76:213-233.

7. Temiz Y, Lovchik RD, Kaigala GV, et al. Lab-on-a-chip devices: How to close and plug the lab? Microelectronic Engineering. 2015;132(25):156175 .

8. Ertl P, Sticker D, Charwat V, et al. Lab-on-a-chip technologies for stem cell analysis. Trends in Biotechnology. 2014;32(5):245-253.

9. Jastrzebska E, Tomecka E, Jesion I. Heart-on-a-chip based on stem cell biology. Biosens Bioelectron. 2016;75:67-81.

10. House DL, Chon CH, Creech CB, et al. Miniature on-chip detection of unpurified methicillin-resistant Staphylococcus aureus (MRSA) DNA using real-time PCR. J Biotechnol. 2010;146(3):93-99.

11. García-Otero N, Barciela Alonso MC, et al. Study of extraction procedures for protein analysis in plankton samples by OFFGEL electrophoresis hyphenated with Lab-on-a-chip technology. Talanta. 2013;115:631-641.

12. Skardal A, Shupe T, Atala A. Organoid-on-a-chip and body-on-a-chip systems for drug screening and disease modeling. Drug Discovery Today. 2016;21(9):1399-1411.

13. Mirasoli M, Guardigli M, Michelini E, et al. Recent advancements in chemical luminescence-based lab-on-chip and microfluidic platforms for bioanalysis. Journal of Pharmacentical and Biomedical Analysis. 2014;87:36-52.

14. Nakanishi W, Minami K, Shrestha LK, et al. Bioactive nanocarbon assemblies: Nan architectonics and applications. Nano Today. 2014;9(3):378-394. 